\title{
Der Personaleinsatz in der schweizerischen
} Entwicklungszusammenarbeit

Engagement du personnel de la coopération technique suisse

Rudolf Dannecker

\section{CpenEdition}

1 Journals

Electronic version

URL: http://journals.openedition.org/sjep/1157

DOI: 10.4000/sjep.1157

ISSN: 1663-9677

Publisher

Institut de hautes études internationales et du développement

\section{Printed version}

Date of publication: 1 janvier 1990

Number of pages: $247-263$

ISSN: 1660-5926

\section{Electronic reference}

Rudolf Dannecker, "Der Personaleinsatz in der schweizerischen Entwicklungszusammenarbeit », Schweizerisches Jahrbuch für Entwicklungspolitik [Online], 9 | 1990, Online erschienen am: 31 März 2013, abgerufen am 08 September 2020. URL : http://journals.openedition.org/sjep/1157 ; DOI : https://doi.org/10.4000/sjep. 1157 


\title{
Der Personaleinsatz in der schweizerischen Entwicklungszusammenarbeit
}

\author{
Rudolf Dannecker
}

Résumé: Engagement du personnel de la coopération technique suisse

Dans la coopération au développement le succès peut parfois largement dépendre du personnel expatrié engagé dans un projet ou un programme et de sa capacité à coopérer avec ses partenaires locaux dans un contexte institutionnel et culturel qu'il ne connaît pas toujours très bien. Cet article présente les fonctions d'un coopérant ainsi que le profil du personnel que la coopération suisse cherche à recruter ainsi que leur évolution en fonction des changements qui interviennent dans la coopération au développement. Toute la séquence des questions liées au personnel de coopération technique est abordée: les motivations du coopérant, les critères et les modalités de recrutement, les rôles complexes du coopérant au carrefour des attentes d'institutions diverses, le retour et la réintégration dans son pays d'origine. L'article se termine par des réflexions sur les perspectives de la coopération technique. 


\section{Elnleltung}

Die Entwicklungszusammenarbeit (EZA) bildet in der Schweiz eine neue und noch relativ junge Komponente unserer Beziehungen mit der Dritten Welt. Trotzdem hat sie in den letzten drei Jahrzehnten bereits eine Reihe von Instrumenten und Arbeitsmethoden mit verschiedenen Zielsetzungen entwickelt. Nicht nur im staatlichen Bereich, sondern auch bei den privaten Entwicklungshilfeorganisationen (oft auch Nicht-Regierungsorganisationen oder abgekürzt NRO's genannt) haben sich verschiedene Konzepte, Priorităten und Ansătze herausgebildet. Zusammen mit dem raschen Anwachsen der finanziellen Mittel für die EZA haben sich auch die verfolgten Strategien stark gewandelt und sind einem permanenten Anpassungsprozess an neue Probleme untenworfen. Hat man vor 30 Jahren noch geglaubt, es handle sich bei der EZA um eine temporäre Aufgabe, so ist heute die Überzeugung gewachsen, dass es sich um eine langfristige Aufgabe der Industrieländer handelt.

Neben Finanzmitteln, Ausrüstungsgütern, Nahrungsmittellieferungen, Stipendien etc. spielt der kurz- oder lăngerfristige Einsatz von Fachleuten eine bedeutende Rolle in der Entwicklungszusammenarbeit. Im folgenden sollen ihre Funktionen und Aufgaben diskutiert, Probleme gezeigt und auch Zukunftsperspektiven erörtert werden. Der Name Entwicklungszusammenarbeit sagt etwas über den Anspruch aus: zusammen etwas erarbeiten, miteinander zu einer Entwicklung beitragen. Der Erfolg dieser Zusammenarbeit und des einzelnen Mitarbeiters vor Ort beruht dementsprechend wesentlich auf der Făhigkeit, mit dem lokalen Partner in einer gemeinsamen Anstrengung Ziele zu erreichen, die zusammen festgelegt werden. Dieses gemeinsame Zusammenarbeiten heisst in der praktisch gelebten Realität sowohl Dialog als auch Auseinandersetzung. Mitarbeiter stehen in einem innen oft fremden Umfeld in einem komplexen Spannungsverhăltnis zwischen verschiedenen Ansprüchen, wie etwa der persönlichen eigenen Motivation, den Zielsetzungen des Projekts und der verschiedenen lokalen Partner, den Vorstellungen der Entwicklungshilfeorganisation im Heimatland etc. Ihre Situation ist deshalb nicht immer ganz klar, oft mit Fragezeichen versehen. So wie sich die Zielsetzungen der Entwicklungszusammenarbeit geăndert haben, hat sich auch das Anforderungsprofil des Feldmitarbeiters gewandelt. Verănderte Zielsetzungen der Projekte und Programme haben zu neuen Anforderungen an das Feldpersonal geführt.

Die Diskussion über Personaleinsătze in der EZA ist nicht frei von "ideologischen Aspekten" und "Modellbildern" des idealen EZA-Mitarbeiters. Verschiedene "Bilder des Entwicklungshilfeexperten" haben die Vergangenheit geprägt wie etwa der "überlegene Fachexperte", der "selbstlose Freiwillige", der "tatkräftige Macher", der "entwicklungspolitisch engagierte Reformer". Die Liste könnte ohne Schwierigkeiten weiter fortgesetzt werden. Diese Bilder haben ihrerseits das Bild der gesamten Entwicklungszusammenarbeit geprăgt und teilweise auch in einer breiteren Öffentlichkeit verfälscht. Die Realităt lăsst sich aber nicht in diesen vereinfachten Bildern fassen; sie ist vielfältiger, differenzierter und komplexer, wie wir im folgenden zu zeigen versuchen. Vergessen wir dabei 
nicht, dass "Experten" oder "Entwicklungshelfer" an sich nicht eine Erfindung der zweiten Hälfte des 20. Jahrhunderts sind, sondern dass seit Jahrhunderten Europäer in verschiedensten Funktionen und mit verschiedenen Zielsetzungen ausgezogen sind in Lănder, die wir heute unter dem problematischen Sammelbegriff der Dritten Welt zusammenfassen. Die ersten waren Entdecker, bald gefolgt von Missionaren. Spăter folgten Hăndler, dann auch Forscher. Mit dem Aufbau der Kolonialherrschaft über weite Gebiete folgten Administratoren und Militärs. Das Bild Europas in der Dritten Welt ist dementsprechend über Jahrhunderte geprăgt worden durch diesen europăischen Einfluss.

Auch heute werden unsere Beziehungen zur Dritten Welt nur zu einem kleinern Teil durch die Entwicklungszusammenarbeit und die Mitarbeiter der EZA beeinflusst und geprăgt. Handelsbeziehungen, Finanzströme, die internationale Wirtschaftspolitik, Investitionen, Tourismus etc. sind oft viel bedeutendere Einflussgrössen als unsere EZA-Programme. In den ărmeren und wenig entwickelten Lăndern bildet die öffentliche Hiffe zweifellos oft einen wichtigen Faktor der wirtschaftlichen und sozialen Entwicklung. Wir düren deshalb aber die EZA und ihre Einflussmöglichkeiten nicht überschătzen und vor allem von ihr nicht die Lösung der Probleme in der Dritten Welt erwarten. Das gleiche gilt für den Feldmitarbeiter der Entwicklungszusammenarbeit. Er ist bei weitem nicht der einzige Vertreter der industrialisierten Welt oder der Schweiz, mit dem Entwicklungsländer und ihre Bevölkerung in Kontakt kommen. Wăhrend die Zahl der Missionare und auch der Entwicklungsexperten abnimmt, haben Kaufleute, Unternehmer, Financiers und vor allem Touristen in den letzten Jahrzehnten die Dritte Welt vermehrt besucht und die gegenseitigen Beziehungen beeinflusst.

\section{Die Organisation der schweizerischen EZA und Ihr Einfluss auf die EZA-Feldmitarbelter}

Gibt es den typischen schweizerischen Feldmitarbeiter? Ist er verschieden von seinen Kollegen aus anderen Industriestaaten? Was gegenüber andern Lănder wie etwa der Bundesrepublik Deutschland oder Frankreich auffăltt, ist die Tatsache, dass in der Schweiz die Feldmitarbeiter nicht von einer einzigen, grossen dominierenden staatlichen Entwicklungsagentur abhăngen. Dies hängt eng mit der internen Struktur unserer bilateralen Entwicklungszusammenarbeit im personellen Bereich zusammen. Schliesst man die grossen Freiwilligenorganisationen in die Betrachtung ein, so zeigt sich, dass von den rund 1000 Feldmitarbeitern nur rund 220 direkt von der Direktion für Entwicklungszusammenarbeit und humanitäre Hilfe (DEH) des Bundes angestellt sind (davon 25 Frauen), wăhrend die übrigen von andern EZA-Organisationen entsandt werden, die oft vom Bund ganz oder teilweise mittinanziert werden. Diese privaten Entwicklungsorganisationen haben ihr eigenes fachliches und entwicklungspolitisches Profil, ein eigenes Beziehungsnetz in der Schweiz mit einer Vielzahl von Mitgliedern und Spendern, mit kirchlichen Organisationen, Ortsgruppen oder anderen Strukturen. Zunehmend ergibt sich zwischen diesen Organisationen eine Ar- 
beitsteilung vor allem durch eine fachliche Spezialisierung, durch eine geographische Konzentration ihrer Unterstützungsmassnahmen sowie durch eine bewusste Wahl der Partnerorganisationen und der Projektansătze.

Der EZA-Feldmitarbeiter hängt somit nicht von einer grossen Organisation ab, sondern von verschiedenen Organisationen mittlerer Grössenordnung. Die Arbeitgeber in der Schweiz sind nicht grosse Entwicklungshilfebürokratien, sondern Institutionen mittlerer Grössenordnung mit je nach Aufgabenbereich 20-40 Mitarbeitern an der Zentrale in der Schweiz und 50-100 ausländischen Feldmitarbeitern (sowie einer grossen Zahl von lokalen Mitarbeitern). Die Beziehung zwischen Feldmitarbeitem und ihrer Zentrale in der Schweiz ist dementsprechend eng und direkt. Die Organisationen ihrerseits sind von ihrer Grössenordnung her überschaubar. Da sie alle ihr eigenes Profil haben, gibt es dementsprechend auch den schweizerischen Modell-Feldmitarbeiter nicht. Die Organisation der schweizerischen EZA entspricht denn auch der auf anderen Gebieten in der Schweiz oft feststellbaren Diversität der Strukturen.

\section{Die verschiedenen Kategorien von Feldmitarbeitern und inre Aufgaben}

Nicht nur in Bezug auf die entsendenden Organisationen, sondern auch in Bezug auf Personalkategorien ergibt sich eine recht grosse Diversität. Auf den ersten Blick gibt es in Bezug auf das Statut zwei Hauptkategorien: die Freiwilligen und die Experten. Wie sehr diese in der Öffentlichkeit oft stark betonte Aufteilung aber weiter differenziert werden muss, zeigt die folgende Zusammenstellung, welche nur einige der Kategorien aufzeichnet, welche in der Realităt vorkommen:

\section{Freiwillige}

- Kirchliche Freiwilligen-Organisationen

- Weltliche Freiwilligen-Organisation

- Langfristfreiwillige

- kurzfristige Katastrophenhelfer etc., etc.

\section{Experten}

- Assoziierte Experten

- Forschungsstipendiaten

- Experten mit begrenzter Vertragsdauer

- Experten mit Langfristvertrag

- Konsulenten (Kurzzeit)

- Administratoren der EZA etc., etc.

In der Realität sind die heute mit der Entwicklungszusammenarbeit direkt oder indirekt verbundenen Kategorien von Mitarbeitern vielfältiger, als hier aufgezeichnet. In der Tat müsste man nicht nur die Feldmitarbeiter in die Betrachtung einbeziehen, sondern auch die in privaten Entwicklungshilfeorganisationen, der Verwaltung, an Hochschulinstitutionen und in andern Bereichen tătigen 
Mitarbeiter, die sich mit Fragen der Entwicklungszusammenarbeit befassen. Auch hier zeigt sich eine breit gefăcherte Diversităt.

Nicht nur haben die EZA-Mitarbeiter verschiedene Personalstatuts, sondern in ihren Tătigkeiten auch recht verschiedene Funktionen und Aufgabenstellungen. In der offiziellen und die Realităt etwas vereinfachenden Sprachregelung der EZA sind die Mitarbeiter im Feldeinsatz zumeist sogenannte "Berater". Dies entspricht oft nicht der von innen erlebten operationellen Realität, in der sie sich unabhăngig von ihrem Statut befinden. Die wirkliche Situation ist komplexer und auch anforderungsreicher als eine reine Beratungsfunktion. Das folgende, vereinfachte Schema versucht in Bezug auf einige ausgewählte Kriterien und Aufgaben - es gibt in der Realität bedeutend mehr - aufzuzeigen, wie komplex die Aufgabenstellungen und wie unterschiedlich die Funktionen im einzelnen sein können. Der z.B. als "technischer Berater" bezeichnete Mitarbeiter kann in der Realităt ganz verschiedene Funktionen ausüben und Verantwortung tragen. In jedem einzelnen Fall kann die Situation verschieden sein und oft auch in verschiedenen Bereichen grosse Unterschiede aufweisen.

Je nach Pflichtenheft, oft aber nach dem im Einsatz nicht im Detail festgelegten Aufgabenkreis, den der Feldmitarbeiter entweder aus eigener Initiative übernimmt oder aber vom Partner mehr oder weniger gezwungenermassen übernehmen muss, variiert dementsprechend sein Aufgaben- und Verantwortungsbereich. Diese Funktionen können nicht immer im voraus klar definiert werden und auch die Erwartungen verschiedener Partner können durchaus verschieden sein. Wăhrend z.B. in der offiziellen Terminologie vor allem aus politischen Gründen immer mehr Berater eingesetzt werden, müssen diese gleichen Berater in der praktischen Projekt- oder Programmrealităt sehr oft inoffizielle oder offizielle operationelle Verantwortung übernehmen oder Kontrollfunktionen ausüben. Vieles hăngt auch vom Vertrauensverhältnis ab, das der Mitarbeiter mit seinen Partnern aufbauen kann. Je grösser dieses Vertrauen, desto stärker auch normalerweise die Tendenz, den Verantwortungsbereich des ausländischen Mitarbeiters zu vergrössern.

Ist somit auf der Ebene des einzelnen Mitarbeiters die Situation schon oft recht komplex, so kann sie im Rahmen eines grösseren Projekts oder Programms von Mitarbeiter zu Mitarbeiter variieren und sogar beim gleichen Mitarbeiter je nach seinen verschiedenen Funktionen unterschiedlich sein. Früher oder später führt ein solches Nebeneinander von unterschiedlichen Funktionen und Aufgaben zu einer Reihe von Fragestellungen und Problemen, mit denen der Feldmitarbeiter konfrontiert wird:

- einmal die Tatsache, dass er "zwei Meister" hat: die lokale Institution, für die er arbeitet und die EZA-Agentur, mit der er einen Anstellungsvertrag hat. Je nach Funktion, Aufgabe und Projektzielsetzung kann im Einzelfall die eine oder andere Komponente stärker wiegen. Die beiden genannten Institutionen haben nicht immer die gleiche Sicht über die Rolle und Funktion des ausländischen Mitarbeiters. Schwierig wird für den Mitarbeiter diese Situation vor allem dann, wenn die Zielsetzungen eines Programms von verschie- 


\begin{tabular}{|c|c|c|c|c|c|c|}
\hline \multicolumn{7}{|c|}{ Kontrollfunktionen - Verantwortllchkelten } \\
\hline \multirow{2}{*}{$\begin{array}{l}\text { Kontrollfunk- } \\
\text { tionen Im } \\
\text { Reale } \\
\text { Funktion }\end{array}$} & \multicolumn{2}{|c|}{ Finanzen } & \multirow{2}{*}{$\begin{array}{l}\text { Tech- } \\
\text { nische } \\
\text { Fragen }\end{array}$} & \multirow[b]{2}{*}{$\begin{array}{l}\text { Allg. } \\
\text { Kontroll- } \\
\text { funktio- } \\
\text { nen }\end{array}$} & \multirow[b]{2}{*}{$\begin{array}{l}\text { Opera- } \\
\text { tionelle } \\
\text { Mitver- } \\
\text { antwor- } \\
\text { tung }\end{array}$} & \multirow[b]{2}{*}{$\begin{array}{l}\text { Gesamte } \\
\text { Operatio- } \\
\text { nelle Ver- } \\
\text { antwor- } \\
\text { tung }\end{array}$} \\
\hline & Lokale & $\begin{array}{l}\text { Auslän- } \\
\text { dische }\end{array}$ & & & & \\
\hline $\begin{array}{l}\text { Reine Berater- } \\
\text { funktion }\end{array}$ & keine & keine & beratend & beratend & keine & keine \\
\hline $\begin{array}{l}\text { Berater mit be- } \\
\text { grenzten techni- } \\
\text { schen Entschei- } \\
\text { dungsfunktionen }\end{array}$ & keine & keine & teilweise & keine & keine & keine \\
\hline $\begin{array}{l}\text { Berater mit finan- } \\
\text { ziellen Kompe- } \\
\text { tenzen }\end{array}$ & keine & $\begin{array}{l}\text { teilweise } \\
\text { verant- } \\
\text { wortlich }\end{array}$ & teilweise & keine & keine & keine \\
\hline $\begin{array}{l}\text { Berater mit Ge- } \\
\text { samtkompetenzen }\end{array}$ & keine & $\begin{array}{l}\text { teilweise } \\
\text { verant- } \\
\text { wortlich }\end{array}$ & teilweise & $\begin{array}{l}\text { verant- } \\
\text { wortlich }\end{array}$ & teilweise & keine \\
\hline $\begin{array}{l}\text { Kodirektor/ } \\
\text { Koverantwortlicher }\end{array}$ & teilweise & $\begin{array}{l}\text { verant- } \\
\text { wortlich }\end{array}$ & $\begin{array}{l}\text { verant- } \\
\text { wortlich }\end{array}$ & $\begin{array}{l}\text { verant- } \\
\text { wortlich }\end{array}$ & teilweise & teilweise \\
\hline $\begin{array}{l}\text { Direktor/Verant- } \\
\text { wortlicher für eine } \\
\text { Aktion }\end{array}$ & $\begin{array}{l}\text { teilweise } \\
\text { verant- } \\
\text { wortlich }\end{array}$ & $\begin{array}{l}\text { verant- } \\
\text { wortlich }\end{array}$ & $\begin{array}{l}\text { verant- } \\
\text { wortlich }\end{array}$ & $\begin{array}{l}\text { verant- } \\
\text { wortlich }\end{array}$ & $\begin{array}{l}\text { verant- } \\
\text { wortlich }\end{array}$ & teilweise \\
\hline $\begin{array}{l}\text { Linienfunktion in } \\
\text { einer lokalen Admi- } \\
\text { nistration/Organi- } \\
\text { sation }\end{array}$ & $\begin{array}{l}\text { ev. ver- } \\
\text { antwort- } \\
\text { lich }\end{array}$ & $\begin{array}{l}\text { ev. ver- } \\
\text { antwort- } \\
\text { lich }\end{array}$ & $\begin{array}{l}\text { verant- } \\
\text { wortlich }\end{array}$ & $\begin{array}{l}\text { verant- } \\
\text { wortlich }\end{array}$ & $\begin{array}{l}\text { verant- } \\
\text { wortlich }\end{array}$ & $\begin{array}{l}\text { verant- } \\
\text { wortlich }\end{array}$ \\
\hline
\end{tabular}

denen Stellen verschieden interpretiert werden oder sogar innerhalb der gleichen Institution von verschiedenen Mitarbeitern unterschiedlich ausgelegt werden;

- im weiteren die Tatsache, dass der Mitarbeiter neben den offiziellen Beratungsfunktionen fast immer auch offiziell deklarierte oder aber faktisch technische und finanzielle Kontrollfunktionen ausübt. Dabei kann es durchaus möglich sein, dass inm solche Funktionen inoffiziell zugewiesen oder aber zugeschrieben werden, auch wenn er sie offiziell nicht ausübt; 
- der Feldmitarbeiter bildet oft das Bindeglied zwischen dem lokalen Partner und ausländischer Hilfe und übt damit eine wichtige und auch notwendige Verbindungsfunktion, aber auch eine Puffer- und Machtfunktion aus. In dieser Doppelfunktion kann er ein betrăchtliches Mass an informellem Einfluss ausüben und massgeblich das Verhalten beider Partner bewusst oder unbewusst steuern. Er kann aber auch in dieser Doppelfunktion in echte Schwierigkeiten und Loyalitătskonflikte geraten;

- der Mitarbeiter steht daneben zudem oft in einem lokalen Spannungsfeld und dies in einem sozioökonomischen Umfeld, dessen Spielregeln, Wertmasstăbe und Verhaltensweisen er nur zum Teil kennt und versteht.

Das Spannungsfeld zwischen dem offiziellen Einfluss (oft schwach) und den informellen Mőglichkeiten der Steuerung (oft recht bedeutsam) ist dementsprechend gross und vielfältig. Dies macht Einsätze in der Entwicklungszusammenarbeit denn auch interessant und bereichernd, aber auch schwierig und anforderungsreich.

\section{Die Motivation der Feldmitarbelter}

Welches sind die Motivationen, die jemanden veranlassen, sich bei einer Entwicklungsorganisation für einen Einsatz zu melden. Entsprechende Erhebungen fehlen, aus unseren Erfahrungen ergeben sich jedoch eine Reihe von regelmässig wiederkehrenden Motivationen. Da ist etwa eine humanitär-ethische Motivation zu nennen, mit oder ohne religiösen Hintergrund. Daneben gibt es beruflich-fachliche Motivationen, das Interesse an einer fremden Kultur, der Wunsch nach Verănderung. Finanzielle Anreize können im Vordergnund stehen, aber auch persönliche Probleme.

Welche Motivationen sind a priori für die EZA wertvoll und gesucht, welche anderseits bilden eine problematische Grundlage für einen Einsatz? Einmal sind verschiedene Motivationen oft vermischt und nicht immer klar auseinander zu halten. Ebenso ist ihre Gewichtung nicht immer einfach. Für verschiedene Aufgaben bildet oft auch eine Mischung verschiedener Motivationen eine gute Grundlage. Problematisch für einen Feldeinsatz sind jedoch alle Motivationen, sofern sie in einer starken Ausprăgung vorliegen, etwa eine stark humanitărmissionarische Ausrichtung, einseitige finanzielle Interessen, alle Fluchtmotivationen (persőnlich, beruflich, zivilisatorisch etc.) oder eine starke Abenteuerlust. Qualităten und Motivationen, die wir demgegenüber in der EZA suchen, sind der Wunsch nach einer neuen beruflichen oder menschlichen Herausforderung, das kulturelle Interesse an einer fremden Welt, die persőnliche Motivation, etwas an die Lossung der Probleme dieser Welt beizutragen etc. Oft suchen wir verschiedene Motivationen, die alle in einem gewissen Mass vorhanden sind. Gefragt sind vor allem Personen mit einer hohen Anpassungsfăhigkeit, einer starken Belastbarkeit und Ausgeglichenheit und mit einer grossen Offenheit gegenüber andern Werten und sozialen Verhaltensweisen. 


\section{Rekrutierung und Selektion}

Die EZA benötigt für ihre Feldeinsătze qualifizierte Fachleute, die über eine gute akademische oder berufliche Ausbildung und mehrere Jahre Berufserfahrung verfügen (Idealalter 30-40 Jahre). Da dies heute in der Schweiz immer schwieriger wird, stellt sich die Frage, wie die Qualităt unserer Rekrutierung beibehalten und wenn möglich erhöht werden kann. Auf Reknutienungsschwierigkeiten und auf einige grundsätzliche Überlegungen zu Rekrutierungsfragen werden wir am Schluss dieser Ausführungen zurückkommen.

Die Frage ist an sich leicht zu beantworten: eine möglichst grosse Zahl von Kandidaten zu haben, die dem für einen Einsatz gewünschten Anforderungsprofil entsprechen. Wie bei jeder Rekrutierung soltten dementsprechend zwei Dinge miteinander in Übereinstimmung gebracht werden können:

- das Anforderungsprofil für einen bestimmten Einsatz

- das Persönlichkeitsprofil des Kandidaten.

In der Praxis ergeben sich allerdings oft eine Reihe von Schwierigkeiten:

- ein Anforderungsprofil aufzustellen, ist nicht einfach, da es oft schwierig ist, eindeutig festzulegen, welche Făhigkeiten und vor allem welche Mischung von Făhigkeiten für einen Einsatz benötigt wird;

- oft werden statt realistischer und möglicher Anforderungen ideale Anforderungsprofile aufgestellt, die Rekrutierungen schwierig machen und die Anzahl möglicher Kandidaten zu stark reduzieren;

- die Beurteilung einer Person im Hinblick auf einen bestimmten EZA-Einsatz ist an sich ein schwieriges Unterfangen, da vor allem das Verhalten in einer fremden Umwelt schwierig zu beurteilen ist;

- die eingesetzten Instrumente bei der Selektion sind noch zu wenig auf spezielle EZA-Einsatzsituationen hin entwickelt worden;

- die spezifische Projektsituation wird oft zu wenig gründlich analysiert und darauf aufbauend ein Anforderungsprofil aufgestellt;

- Personalentscheide werden zu stark gefühlsmässig getroffen und zu wenig abgestützt auf fach- und sachbezogene Überlegungen.

Die Selektion ist deshalb wichtig, weil Misserfolge im Einsatz zumeist sowohl für die betroffenen Projekte und Institutionen wie auch für die Betroffenen selbst zu schwierigen Situationen führen, und eine vorzeitige Rückkehr aus einem Einsatz schwieriger zu verkraften ist, als ein beruflicher Misserfolg in der Schweiz.

Ein ausgebautes Instrumentarium für die Selektion von Feldmitarbeitern ist heute vorhanden und wird auch normalerweise eingesetzt, wie die folgende Liste der von professionellen EZA-Organisationen benutzten Instrumente zeigt:

- eine eingehende Analyse des bisherigen Lebenslaufes

- eine Selbsteinschätzung der Kandidaten auf ihre Eignung 
- strukturierte Gespräche mit mehreren Personen

- Einholen von Referenzen (nur mündlich und mit gezielten Fragestellungen)

- Gespräch und Beurteilung durch Drittpersonen

- graphologische Gutachten, etc.

Diese Instrumente werden je nach Schwierigkeit eines Postens selektiv oder gesamthaft eingesetzt. Wichtig ist dabei nicht die Suche nach dem idealen Mitarbeiter für die Entwicklungszusammenarbeit, sondern die Selektion des mehr oder weniger geeigneten Kandidaten für eine bestimmte Aufgabe zu einem bestimmten Zeitpunkt in einer bestimmten Situation. Zweifellos sind die Anforderungen für Einsătze in der Entwicklungszusammenarbeit recht verschiedenartig. Trotzdem ergeben sich eine Reihe von Kriterien, die vor allem von Bedeutung sind, wie z.B. berufliche Anforderungen, die spezifischen Anfordenungen der Funktion/Aufgabe, die in einem Projekt bestehende Team- und Partnersituation. Wichtig ist auch die institutionelle Position und Funktion, die zu besetzen ist, die lokale Lebenssituation sowie eine Reihe anderer Kriterien.

Interessant ist in der praktischen Rekrutierungs- und Selektionssituation, dass zu wenig zwischen lernbaren Fähigkeiten (Sprache, Spezialkenntnisse etc.) einerseits und nicht kurzfristig beeinflussbaren Făhigkeiten (Grundausbildung, Kommunikationsfähigkeit etc.) unterschieden wird. Ebenso bildet die Definition von Anforderungsprofilen (wer wird für eine Aufgabe wirklich benötigt) noch einen Schwachpunkt in der EZA. Wie bereits erwähnt, wird noch zu lange nach dem idealen Kandidaten gesucht (statt nach dem möglichen). Ein auf den ersten Blick mittelmässiger Kandidat mit breitem Horizont und Anpassungsfähigkeit wird im praktischen Projekteinsatz oft erfolgreicher sein, als der zu eng spezialisierte Fachmann, der dann den oft unvorhersehbaren Anforderungen im Einsatz nicht gewachsen ist. Zu hohe Anforderungsprofile führen nach längerer erfolgloser Suche dann zum Teil zu Panikrekrutierungen, welche problematische Folgen haben können. Oder aber es wird ein einzelnes Kriterium überbewertet (z.B. Landes- und Sprachkenntnisse, entwicklungspolitische Überzeugung, Fachkenntnisse etc.), ohne entsprechende Berücksichtigung weiterer, ebenfalls relevanter Kriterien. Und schliesslich wird immer mehr Drittwelterfahrung bei Einsătzen vorausgesetzt, was die mögliche Zahl von Kandidaten stark reduziert, aber nicht immer die erwarteten Resultate bringt.

\section{Probleme beim Feldeinsatz und bel der Rückkehr}

Wo liegen die hauptsăchlichen Schwierigkeiten beim Einsatz unserer Mitarbeiter im Feld? Vorerst ist festzuhalten, dass es natürlich ganz verschiedene Einsatzbedingungen gibt und die einzelnen Situationen dementsprechend stark variieren können. Die Anforderungen zwischen dem Leben in einer Hauptstadt oder in einer abgelegenen lăndlichen Gegend, oder die Tătigkeit als Berater in einer Verwaltung oder als Mitarbeiter einer operationellen Projektequipe weisen grosse Unterschiede auf. Dementsprechend sind auch die Probleme recht verschie- 
dener Natur. In der Praxis können sie in einige Kategorien klassiert werden, nămlich in Probleme auf operationeller Ebene, im menschlich-kulturellen $\mathrm{Be}$ reich sowie auf beruflich-fachlicher Ebene.

Oft sind unsere Mitarbeiter nicht besonders an administrativen Fragen interessiert, für die sie zudem nicht immer eigene Erfahrungen mitbringen. Ihr Interesse liegt vielmehr auf technischem und operationellem Gebiet, bei den sozialen oder fachlichen Zielsetzungen. Administrative Reglemente, Buchhaltungsvorschriften, Richtlinien für Dienstfahrzeuge oder andere Weisungen sind an sich nicht besonders interessant. Der im Einsatz stehende Forstingenieur oder Agronom, Soziologe oder Bauingenieur wird diese Richtlinien oft als unnötigen administrativen Autwand empfinden. Dahinter steckt jedoch auch ein tiefergreifendes Problem der Entwicklungszusammenarbeit: Wie weit ist es überhaupt möglich für die verschiedensten Situationen zentrale Weisungen und Reglemente aufzustellen und wie weit muss nicht eine möglichst grosse Flexibilităt in administrativen Belangen Ziel für eine angepasste Entwicklungszusammenarbeit bleiben. Ein entscheidender Schritt in diesem Bereich hat die DEH durch den Einsatz von Aussenrevisoren in Schwerpunktländern unternommen, die die Projektmitarbeiter im administrativen Bereich beratend unterstützen.

Auf operationellem Gebiet geht es oft um Fragen der Zielsetzungen eines Projekts, um Konzepte und Vorgehensmethoden. Neben diesen mehr grundsătzlichen Fragestellungen kann es sich aber auch um materielle oder finanzielle Vorteile handeln, welche das Projekt auf Grund seiner Aktivităten verschaffen kann (Besetzung von Stellen, Vergebung von Aufträgen etc.) und die zu schwierigen Situationen für Feldmitarbeiter führen können.

Im menschlichen Bereich können sich Probleme innerhalb einer Equipe von Auslăndern ergeben, aber auch Spannungen mit lokalen Partnern. Persönliche und familiäre Probleme des einzelnen Mitarbeiters sowie die Isolation in einem fremden Umfeld sind weitere Problembereiche. Gerade in diesem persönlichen und zwischenmenschlichen Bereich kommt der richtigen Selektion und Vorbereitung der Mitarbeiter eine grosse Bedeutung zu.

Auf beruflichem Gebiet schliesslich bildet oft die berufliche Isolation eine Belastungsprobe. Da die beruflichen Anfordenungen im Einsatz in der Dritten Welt zumeist anders liegen als in den Industriegesellschaften (andere Technologie, andere Methoden etc.), kann der fehlende berufliche Kontakt und Austausch mit aktuellen Berufsfragen in einer Industriegesellschaft zu Befürchtungen führen, bei einer Rückkehr den Anschluss zu verlieren.

Zwischen Ansprüchen des Partners und Erwartungen der Schweiz befindet sich somit der Feldmitarbeiter oft in einer komplexen Situation, in einem Geflecht von Beziehungen, welche seine Orientierung erschweren und zur Verunsicherung beitragen.

Diese Ausführungen zeigen, dass die an den Auslandmitarbeiter der EZA gestellten Anforderungen oft ausserordentlich vielfăltig sind und eine grosse Breite aufweisen. Nicht nur soll er gleichzeitig ein guter Planer und Organisator sein, sondern auch über die notwendigen fachlichen Qualifikationen und die Sensibilităt gegenüber einer fremden Kultur verfügen. Man kann sich fragen, 
wie weit solche Anforderungsprofile nicht zu sehr einem Wunschdenken entsprechen und wir unsere Vorstellungen in Bezug auf die realen Möglichkeiten nicht etwas anpassen müssen.

Wenn wir hier von Problemen und Schwierigkeiten sprechen, so dürfen wir nicht vergessen, dass in der grossen Mehrzahl die Einsătze erfolgreich verlaufen, auftretende Schwierigkeiten gelöst werden und unsere Mitarbeiter es oft bedauern, nach einigen Jahren wieder in die Schweiz zurückkehren zu müssen. Gerade diese Rückkehr kann denn auch zum Problem werden, insbesondere für Mitarbeiter, die lăngere Zeit in der Dritten Welt im Einsatz standen. Schwierigkeiten können sich dabei auf verschiedenen Ebenen ergeben:

- Die berufliche Reintegration wird erschwert, da durch einen längern Aufenthalt in der Dritten Welt der fachlich-berufliche Anschluss verloren gegangen ist.

- Die persönliche Reintegration; d.h. das sich Zurückgewöhnen in eine industrielle Gesellschaft (beruflich, familiär, gesellschaftlich etc.) ist oft mit Problemen verbunden.

- Das Erleben anderer Kulturen führt zu einer Auseinandersetzung mit unserer Gesellschaft und ihren Werten allgemein und zum Nachdenken über grundsătzliche Fragestellungen in unserer eigenen Entwicklung.

Gegenwärtig werden diese Fragen der Rückkehrerbetreuung in der DEH eingehend diskutiert und ein neues Konzept der Beratung ausgearbeitet, das im Verlauf von 1990 eingeführt werden soll.

\section{Die praktischen Probleme bel der Rekrutierung von EZA-Mitarbeitern in der Schweiz}

Vorerst ist festzhuhalten, dass die Zahl der Feldmitarbeiter in der EZA seit einigen Jahren abnehmende Tendenz hat. Dies aus verschiedenen Gründen:

- Für viele Aufgaben gibt es heute ausgebildete Kader in der Dritten Welt.

- Vermehrt werden auf Spezialgebieten kurzfristig Konsulenten an Stelle von Langfristmitarbeitern eingesetzt.

- Die Kosten für einen Einsatz sind hoch und lassen sich nur vertreten bei entsprechend wichtigen Führungs-, Planungs- und Organisationsfunktionen.

Trotz diesem Rückgang ergeben sich in der Schweiz gegenwärtig Schwierigkeiten, das für Feldeinsătze benötigte Personal zu finden. Andererseits besteht jedoch eine grosse Nachfrage nach Einsatzmöglichkeiten in der Dritten Welt. Allerdings kommen von 100 Anfragen über Einsatzmöglichkeiten nur etwa 2030 für einen Feldeinsatz überhaupt in Frage auf Grund beruflicher und fachlicher Qualifikationen und davon können vielleicht 5 dann wirklich in einen Einsatz ausreisen. In der Mehrheit der Fälle entsprechen die beruflichen Anforde- 
rungen (Berufsrichtung, Ausbildungsniveau und Erfahrung), nicht den Bedürfnissen im Feld, was zu der paradoxen Situation führt, dass zwar zu viele Interessenten für offene Stellen vorhanden sind, aber trotzdem nicht alle Stellen rechtzeitig zufriedenstellend besetzt werden können.

Welches sind die Gründe für diese wenig zufriedenstellende Situation, die sich in den letzten Jahren noch verschärtt hat. Wir haben keine gesicherten Untersuchungsergebnisse, die uns weiterhelfen könnten und sind deshalb auf $\mathrm{Hy}$ pothesen angewiesen:

- Eine wichtige Rolle spielt zweifellos die Situation des Arbeitsmarktes in der Schweiz mit Vollbeschäftigung und akutem Arbeitskräftemangel auf einer Reihe von Gebieten. Es ist gegenwärtig in der Schweiz schwierig, das von der EZA geforderte Anforderungsprofil auf dem Arbeitsmarkt zu finden: nämlich fachlich gut qualifizierte Berufsleute mit unversitärer oder anderer höherer Fachausbildung, einer Berufserfahrung von 5-10 Jahren und dem gesuchten menschlichen Anforderungsprofil.

- Viele der Interessenten, die sich unmittelbar nach Abschluss inrer Ausbildung für einen Einsatz interessieren, (und dann dafür zumeist noch zu jung sind) sind im gesuchten Idealalter von 28-35 Jahren aus familiären und beruflichen Gründen weniger bereit, ihre soeben begonnene Karriere zu unterbrechen und einige Jahre in der Dritten Welt zu verbringen.

- Oft ist bei einem Einsatz insbesondere in ländlichen Gebieten nur für einen Ehepartner eine Anstellung möglich. Normalerweise handelt es sich dabei um den Ehemann. Da heute Frauen vermehrt enwerbstätig sind und ebenfalls ihre persönliche Laufbahnplanung haben, beeinträchtigt ein solcher Einsatz vor allem die Enwerbstätigkeit und die Laufbahn der Frau. Der Entscheid, ob eine Ausreise in Frage kommt oder nicht, hängt deshalb vermehrt weitgend von den Frauen ab. Da wir für eine Reihe von Einsätzen in erster Linie verheiratete Mitarbeiter suchen, beschränkt dies die Möglichkeiten.

- Für Frauen sind die Einsatzmöglichkeiten zumeist beschränkt und dies aus verschiedenen Gründen. Einmal sind verschiedene Schwerpunktbereiche der schweizerischen Entwicklungszusammenarbeit schon vom beruflichen Profil in der Schweiz her zahlenmässig stark von Männern dominierte Berufsrichtungen wie etwa Agronomen und Forstingenieure, sowie z.B. technische Berufe im Ingenieur- und Baubereich. Begrenzte Möglichkeiten ergeben sich aber oft auch durch Hindernisse in der Dritten Welt, in dem viele Funktionen in der Verwaltung den Männern vorbehalten sind und der Einsatz von Frauen aus gesellschaftlichen, sozialen oder religiösen Vorstellungen auf Schwierigkeiten stösst.

- Die EZA kann nur einer beschränkten Anzahl von Mitarbeitern eine längerfristige Sicherheit anbieten - für die grosse Mehrzahl der Ersteinsätze handelt es sich um verlängerbare Zwei- bis Dreijahresverträge. Die meisten Feldmitarbeiter machen 4-8 Jahre (einen bis zwei Einsätze) in der EZA, bevor sie sich wieder in der Schweiz reintegrieren. Eine sichere Karriere gibt es in der EZA nicht oder nur für einen beschränkten Teil der Mitarbeiter. 
- Eine gewisse Ernüchterung über die EZA und ihre Resultate hat wohl das Interesse an Einsătzen allgemein etwas vermindert.

- Die Anforderungen in Bezug auf Ausbildung weisen weiterhin steigende Tendenz auf, was die Schwierigkeiten der Rekrutierung erhöht. Gefragt ist heute der breit ausgerichtete Fachmann und weniger der Pionier der ersten Jahrzehnte der EZA.

- Heute ist es für den kaufkraftstarken Schweizer einfach geworden, auch ohne EZA in die Dritte Welt zu reisen. Das Fremdartige der Dritten Welt hat seine Attraktion teilweise eingebüsst.

- Schliesslich müssen wir uns auch die Frage stellen, ob sich die EZA richtig und gut auf dem schweizerischen Stellenmarkt verkauft. Es scheint vielmehr, dass es den EZA-Organisationen nicht gelungen ist, ein klares Profil und Bild des gesuchten Mitarbeiters und der interessanten Seiten eines Einsatzes einer breiten Öffentlichkeit zu vermitteln.

Was unternehmen schweizerische EZA-Organisationen angesichts dieser Rekrutierungsschwierigkeiten? Einmal wird als Sofortmassnahme auf andere europăische Lănder ausgewichen (Deutschland, Frankreich, England, Holland), wo es auf Grund der Arbeitsmarktsituation bedeutend einfacher ist, erfahrene Fachleute mit Drittwelterfahrungen zu rekrutieren. Die Zahl der Feldmitarbeiter mit auslăndischer Nationalităt weist dementsprechend steigende Tendenz auf. Gegenwărtig liegt dieser Anteil je nach Organisation bei durchschnittlich 20-30 Prozent.

Die Frage nach Verbesserung der Rekrutierungssituation hat in den letzten Jahren die schweizerischen EZA-Organisationen zunehmend beschäftigt und ist sowohl an verschiedenen internen Tagungen in einzelnen Organisationen wie auch an gemeinsamen Seminarien eingehend diskutiert worden. Aus diesen Diskussionen ergaben sich eine Reihe von Prioritäten für die Zukunft:

- Einmal besteht Übereinstimmung, dass weiterhin EZA-Mitarbeiter einem Bedürfnis entsprechen und dies auch lăngerfristig. Die Zahl dürtte weiterhin leicht abnehmen, die Anforderungen dagegen steigende Tendenz aufweisen.

- Deutlich wurde auch der Wunsch der grössern EZA-Organisationen, vermehrt untereinander im Personalsektor zusammenzuarbeiten. So sollen u.a. der Wechsel von einer Organisation zur andern durch entsprechende Massnahmen erleichtert werden.

- Es müssen auch vermehrt die zunehmenden lokalen Ressourcen ausgeschöpft werden durch Anstellung von lokalem Projektpersonal und lokalen Konsulenten. Eine Reihe von flankierenden Massnahmen sind dafür notwendig wie gezielte Personalförderungsmassnahmen, Aufbau einer lokalen Konsulentendatei, Förderung von gemeinsamen Missionen zwischen auslăndischen und lokalen Konsulenten etc.

- Projekte und Programme sind so an lokale Verhältnisse anzupassen, dass der Einsatz von Auslandspersonal klein gehalten werden kann. Dies kann 
einen wichtigen Einfluss auf Projektkonzepte und Realisierungsrhythmus der Programme ausüben.

- Die Rahmenbedingungen im Feld sind so zu wăhlen, dass qualifiziertes Auslandspersonal gefunden werden kann (Schulen für Kinder, Klima, Unterkunft, etc.).

- Und schliesslich ist man überzeugt, dass das Rekrutienungsumfeld in der Schweiz durch geeignete Massnahmen zu verbessern ist; d.h. dass eine Imageverbesserung der EZA durch geeignete Massnahmen notwendig ist.

\section{Einige grundsătzliche Fragestellungen zum Experteneinsatz}

Im Zusammenhang mit unseren Ausführungen zum Experteneinsatz ergeben sich eine Reihe von grundsătzlichen Fragestellungen. Vermehrt wird heute gerade in EZA-Kreisen die Frage gestellt, ob in der EZA in der Zukunft überhaupt noch Experten eingesetzt werden sollen und ob wir nicht vom "Expertenprojekt" wegkommen und zu einer neuen Form von Projekten übergehen sollten, die ohne ausländische Expertise auskommen. Meiner Auffassung nach ist diese Fragestellung falsch. Auszugehen ist nicht von der oft entwicklungspolitischen oder entwicklungsphilosophischen Frage, ob auslăndische Experten an sich in Projekten notwendig sind oder nicht, sondern vielmehr von den Projekten, ihren Inhalten und ihren Zielsetzungen. Welche Massnahmen sind zu treffen, um die Zielsetzungen eines Programmes zu erreichen? Ist dies ohne ausländische Experten möglich? Zu berücksichtigen sind dabei aber nicht nur fachliche $\mathrm{Ge}$ sichtspunkte, sondern auch konzeptionelle, politische, gesellschaftliche und institutionelle Überlegungen, um nur einige zu nennen. Sicher muss es ein Ziel sein, Programme ohne personelle ausländische Hiffe zu realisieren. Sofem dieses Ziel jedoch einen zu hohen Stellenwert erhălt, kann es entweder zur Nichtrealisierung einer ganzen Reihe von Programmen führen oder aber zu Misserfolgen in der Projektarbeit. Eine differenzierte, in jedem einzelnen Fall abwägende und überlegende Haltung ist deshalb notwendig. Anderseits sind die Tendenzen in der schweizerischen Entwicklungszusammenarbeit in den letzten zehn Jahren eindeutig: Die Zahl der Experten in Projekten nimmt ab, die Anforderungen an die einzelnen Einsătze nehmen zu und anderseits steigt der Personalbestand in den Koordinationsbüros rasch an. Neben den Koordinatoren und Administratoren werden dort immer hăufiger auch Fachspezialisten für klar umrissene Fachbereiche eingesetzt. Diese Tendenzen werden sich voraussichtlich weiterhin verstärken.

Im Zusammenhang mit dem Experteneinsatz ergeben sich aber auch eine Reihe von weiteren grundsătzlichen Fragestellungen:

- Unserem Anspruch - und oft auch dem Wunsch des Partners - auf Mitsprache oder Mitsteuerung der Programme steht das Ziel gegenüber, mit selbständigen Partnern zusammenzuarbeiten, die ihre Ziele in eigener Verant- 
wortung setzen und ihre Programme in ihrem Rhythmus realisieren. In diesem Zielkonflikt ist es wichtig, dass in jedem einzelnen Programm klare Priorităten zusammen mit dem Partner gesetzt werden und im einzelnen festgelegt wird, in welchem Bereich welche Zielsetzung Priorităt hat. Eine differenzierte Betrachtungsweise ist dabei notwendig, und es wird sich in der konkreten Projektsituation enweisen, dass für gewisse Bereiche die Selbstăndigkeit des lokalen Partners, für andere wiedenum unsere aktive Mitbestimmung Priorităt hat.

- Wie handhaben wir "die Kontrolle" in expertenfreien Projekten in Bezug auf Berichterstattung, Abrechnungen, Evaluation und Monitoring des Projektfortschrittes? Wie lösen wir die Problematik zwischen unseren administrativen, operationellen und finanziellen Kontrollansprüchen und eventuell anders gelagerten Bedürnissen und Erwartungen unserer Partner? Wie weit gehen wir mit unserer Forderung nach Erfüllung unserer Ansprüche? Gerade auch "ohne Experten" stellt sich die Frage, wie weit wir im "Dialog" mit unserem Partner gehen kőnnen oder wollen. Wo liegen die Grenzen eines solchen Dialogs? Wie weit z.B. wollen und vor allem können wir unseren Partner begleiten in politischen delikaten Situationen?

- Wie weit wird beim Expertenprojekt der Informationsfluss vom Partner zur EZA-Zentrale durch den "Experten" bewusst oder unbewusst gefiltert? Umgekehrt aber ist die Frage auch legitim: wie weit erfolgt eine ebenso starke Filterung durch den lokalen Partner gegenüber dem Geldgeber?

- Ohne "Experten" wird die Beurteilung der Situation vor Ort schwieriger. Die DEH selbst verfügt über entsprechende Infrastrukturen (Koordinationsbüros), die in Zukunft noch vermehrt ausgebaut werden, insbesondere wenn die Zahl der "expertenlosen Projekte" zunimmt. Steigt damit nicht die Abhăngigkeit schweizerischer EZA-Organisationen von diesen Koordinationsbüros der DEH, die mit ihren Dienstleistungen und inrem lokalen Beziehungsnetz steigenden Einfluss ausüben? Und ergibt sich dann nicht auch eine steigende Abhăngigkeit lokaler Partner von diesen Koordinationsbüros, die als Vertreter der schweizerischen Entwicklungszusammenarbeit über eine recht betrăchtliche Machtfülle und hohe Steuerungsmöglichkeiten verfügen? Die oft angeführte Partnernăhe des Koordinationsbüros ist zweifellos oft ein Vorteil - ist es aber nicht auch eine Gefahr? Anstelle der Gefahr einer Dominanz durch den "Experten" tritt die Gefahr einer Dominanz durch den Mitarbeiter der Zentrale auf Kurzbesuchen oder die Macht des Koordinationsbüros.

- Es scheint mir auch schwierig, die Problematik des "Entwicklungshelfers" zu trennen von der Problematik der Entwicklungszusammenarbeit ganz allgemein. Ist die Annahme richtig, dass eine Unterstützung ohne auslăndisches Personal an sich besser und angepasster ist als Unterstützungsmassnahmen mit auslăndischem Personal? Ist eine solche Hilfe aber wirklich nicht interventionistisch, wie oft behauptet wird? Handelt es sich dabei nicht um eine intellektuelle Fiktion, die einer konkreten Analyse in der Praxis nicht standhätt? 
- Besteht nicht die Gefahr, dass mit starkem lokalem Personaleinsatz zwar die sozio-ökonomische Projektintegration gefördert, dadurch aber auch institutionelle, fachliche, methodische und personelle Schwăchen verstărkt oder zumindestens nicht gelöst werden? Geht uns damit nicht oft auch eine wichtige Antenne zur Analyse der eigentlichen Probleme einer Partnerorganisation verloren?

- Der EZA-Mitarbeiter ist meiner Auffassung nach ein Instrument der EZA neben vielen anderen: er ist nicht an sich gut oder unangepasst. Wichtig ist, dass in jedem einzelnen Fall die Bedürnnisse mit dem Partner auf Grund seiner Făhigkeiten und der Projektzielsetzungen abgesprochen werden. Das "personelle Element" in der EZA hat meines Erachtens jedoch auch in Zukunft eine bedeutsame Rolle zu spielen. Zentral dabei ist die Auseinandersetzung zwischen verschiedenen Kulturen und ihren Werten. Dieser Dialog kann in verschiedenen Formen vor sich gehen, aber er soltte immer auch zum Inhalt haben, dass wir dabei zusammen mit dem Partner Fragen stellen. Deshalb solte in Zukunft nicht die Frage im Vordergrund stehen, ob noch ausländisches Personal in Entwicklungsprogrammen eingesetzt wird, sondern dass wir uns vermehrt mit der Frage auseinandersetzen, welches Anforderungsprofil diese Mitarbeiter haben sollten, welchem Anforderungsprofil lokale Mitarbeiter entsprechen sollten und welche Konsequenzen "expertenlose Projekte" oder "Expertenprojekte" haben, wo sie eine Chance und wo sie vielmehr eine Gefahr für das angestrebte Entwicklungsziel darstellen.

Wie wir festgestellt haben, gehen die gegenwärtigen Tendenzen eindeutig in Richtung auf den Einsatz von mehr Lokalpersonal, mehr Kurzfristeinsätzen von ausländischen Konsulenten, zu einem Abbau von ausländischem Personal in Langfristeinsätzen in Projekten und zu einem personellen Ausbau der Koordinationsbüros. Ist dies langfristig die richtige Lösung?

Richtig daran ist, dass in Zukunft eine "zunehmende Professionalität in der EZA" notwendig sein wird und dies sowohl für Einsătze in Projekten wie auch in Koordinationsbüros. Professionalisierung in der EZA bedeutet die Gesamtheit der Fähigkeiten zur Suche nach und Ausarbeitung von Problemlösungen mit unseren Partnern, die wirtschaftlich, ökologisch und sozial-gesellschaftlich nachhaltige Wirkung zeitigen, d.h. vom gesamten Umfeld mitgetragen werden. Diese Professionalităt ist eine ehrgeizige Zielsetzung und sie verlangt eine permanente Aus- und Weiterbildung in drei Bereichen, nämlich im fachlich-methodischen Bereich, in der Persönlichkeitsbildung und im einsatzspezifischen $\mathrm{Be}$ reich, d.h. in der Vorbereitung auf eine praktische Aufgabe in der EZA. Diese Professionalität ist Voraussetzung für eine Laufbahn in der Entwicklungszusammenarbeit der neunziger Jahre.

Ausgehend von diesen Überlegungen ergeben sich eine Reihe von Folgerungen für den Einsatz von "Experten":

- die schweizerische EZA muss fachliche Schwerpunkte bilden auf ausgewählten sektoriellen Bereichen, sowie innerhalb dieser Bereiche auf Bera- 
tung, Organisationsentwicklung, Planung und Evaluation, Koordination und Politikdialog.

- Die Bildung eines professionellen Kaders sowohl von langfristigen Mitarbeitern wie auch von Kurzfristkonsulenten ist notwendig, wobei die personellen Ressourcen zwischen DEH, Hiffswerken und anderen Organisationen inkl. privaten Unternehmen besser gegenseitig genutzt und auch ausgetauscht werden sollten.

- Die Projektauswahl, die Projektkonzepte und die Projektstandorte müssen vermehrt den in der Schweiz vorhandenen personellen Ressourcen angepasst werden.

- Ein angepasstes System von Aus- und Weiterbildung ist notwendig, um die Qualităt dieser Professionalisierung zu erhöhen.

Für diese Personalpolitik der neunziger Jahre in der EZA müssen die DEH, schweizerische Hilfswerke, die offentliche Verwaltung und private Firmen zusammenarbeiten, um eine optimale Nutzung der in der Schweiz vorhandenen personellen Ressourcen zu erreichen. Ein erster wichtiger Schritt in dieser Richtung bildet die Gründung der neuen Stiftung für Berufsinformation und Rückkehrerberatung, die anfangs 1990 durch die DEH zusammen mit anderen EZA-Organisationen und dem schweizerischen Verband für Berufsberatung erfolgte. 